\title{
Autonomous Vehicles: An Analysis Both on Their Distinctiveness and the Potential Impact on Urban Transport Systems
}

\author{
Alessandro Severino ${ }^{1, *(\mathbb{D})}$, Salvatore Curto ${ }^{2} \mathbb{D}$, Salvatore Barberi ${ }^{2}$, Fabio Arena ${ }^{2}$ and Giovanni Pau ${ }^{2} \mathbb{D}$ \\ 1 Department of Civil Engineering and Architecture, University of Catania, 95123 Catania, Italy \\ 2 Faculty of Engineering and Architecture, Kore University of Enna, 94100 Enna, Italy; \\ salvatore.curto@unikorestudent.it (S.C.); salvatore.barberi@unikore.it (S.B.); fabio.arena@unikore.it (F.A.); \\ giovanni.pau@unikore.it (G.P.) \\ * Correspondence: alessandro.severino@unict.it
}

Citation: Severino, A.; Curto, S.;

Barberi, S.; Arena, F.; Pau, G.

Autonomous Vehicles: An Analysis Both on Their Distinctiveness and the Potential Impact on Urban Transport Systems. Appl. Sci. 2021, 11, 3604 https://doi.org/10.3390/app11083604

Academic Editor: Luís Picado Santos

Received: 14 March 2021

Accepted: 14 April 2021

Published: 16 April 2021

Publisher's Note: MDPI stays neutra with regard to jurisdictional claims in published maps and institutional affiliations.

Copyright: (C) 2021 by the authors. Licensee MDPI, Basel, Switzerland. This article is an open access article distributed under the terms and conditions of the Creative Commons Attribution (CC BY) license (https:/ / creativecommons.org/licenses/by/ $4.0 /)$

\begin{abstract}
Autonomous driving is a technological innovation that involves the use of Artificial Intelligence (AI) in the automotive area, representing the future of transport and whose applications will influence the concept of driving and many other features of modern society. Indeed, the introduction of Autonomous Vehicles (AVs) on the market, along with the development of related technologies, will have a potential impact not only on the automotive industry but also on urban transport systems. New mobility-related businesses will emerge, whereas existing ones will have to adapt to changes. There are various aspects that affect urban transport systems: in this work, we highlight how road markings, intersections, and pavement design upgradings have a key role for AVs operation. This work aims to describe how contemporary society will be influenced by Autonomous Vehicles' spread in regard to urban transport systems. A comprehensive analysis of the expected developments within urban transport systems is hereby presented, and some crucial issues concerning benefits and drawbacks are also discussed. From our studies, it emerges that the detection performed by vehicles is mainly affected by road markings characteristics, especially at intersections. Indeed, the need for a new cross-sections type arise, since vehicles wandering phenomena will be reduced due to AVs position-keeping systems.
\end{abstract}

Keywords: autonomous vehicles; intelligent transportation system; road safety; urban transport system; artificial intelligence

\section{Introduction}

In modern era, safety aspects have gained considerable importance within the transportation industry. As a consequence of significantly increasing traffic volumes and in association with the growing popularity of mass transportation, in recent years, the number of vehicle accidents has increased leading to greater consequences on people's quality of life but also having an effect on government financial expenses.

In 2016, The World Health Organization estimated that the number of deaths related to road accidents was over 3.400 per day, with associated cost having an impact of nearly the $3 \%$ on the World's Gross Domestic Product. One of the most recent solutions to ease and improve traffic operations is the introduction of Autonomous Vehicles (AVs), which consists of special vehicles operated automatically by electronic systems designed to achieve high-performance levels with superior accuracy, greater than human capabilities.

A reduction in the number of accidents and related financial losses is expected as traffic becomes regulated by electronic devices and computer algorithms, reducing the probability of accidents caused by human mistakes. In this sense, an example of a commonly adopted device is the Adaptive Cruise Control (ACC), a driving assistant system that allows the driver to automatically cruise its vehicle according to the preceding user's 
behavior. Cooperation between AVs leads to interconnected systems called "Connected and Automated Vehicles technology" (CAVs) [1].

In recent years, several car manufacturers such as Tesla and Audi have launched on the market autonomous vehicles for private use. A significant spread can also be noted within the public transport sector, with the more frequent employment of autonomous minibuses with a maximum capacity of 15 passengers, mostly powered by electric engines; for example, Navya or Easymile are some of the companies providing autonomous shuttles for various environments such as parks, universities, hospitals, and airports (these types of shuttles operate with a maximum speed of $25 \mathrm{~km} / \mathrm{h}$ and with a maximum slope of $12 \%$. Speaking of safety, middle- and low-income countries should deserve a particular focus. Indeed, it has been noted that they are more susceptible to road accidents with all the related consequences, possibly due to the reduced availability of advanced technological devices for roads and vehicles. These countries represent $84 \%$ of the global population and, despite the low number of registered vehicles, $92 \%$ of road accidents have fatal consequences [2].

Eventually, this work presents an analysis of AVs' effects on urban transport systems. An overview about AVs' peculiarities is reported in Section 2, introducing the typical hardware, some autonomous driving classification, and typical operational tools and discussing also the expectations in terms of economic impact. As AVs spread into common use, many arising problems must be solved in order to improve transport safety; Section 3 describes the state of the art presenting some of the principal AV benefits and drawbacks, highlighting how the urban transport system could receive potential feedbacks in terms of emissions, leading to positive effects on public health also considering the recent COVID-19 pandemic. Although AVs are equipped with several high-level sensors, both current urban transport systems and road infrastructure demand upgrades to guarantee an efficienct use and safe journeys. In this sense, Section 4 and analyze several AV critical issues, two of which influence both infrastructure design and operation phases. Finally, Sections 5 and 6 summarize and discuss all the treated topics, suggesting observations and proposing new ideas about the spread of AVs into urban transport systems.

\section{Background}

The introduction of AVs within the transport industry has become gradually effective with time, with an acceleration in the last decade. Their operations are affected by several factors, depending mainly on elements such as type of installed devices, scenarios, and country legislations; since these aspects affect the specific level of autonomy of vehicles, they heavily influence the spread of AVs.

Depending on tools and vehicles equipment and according to the classification provided by SAE (Society of Automotive Engineers), it is possible to divide autonomous vehicles into two classes, each characterized by six levels of automation: the first class, equipped with ADS (Automated Driving Systems) which offers autonomous vehicle performances, and the latter equipped with tools for driver assistance only. Eventually, ADS sensors and the aforementioned tools allow vehicles to establish a V2I, V2V, and V2X communication that enables environment detection and, consequently, autonomous driving maneuvers. In addition to these technological systems, autonomous vehicles are equipped with LiDAR, GPS, cameras, and sensors whose performances have recently achieved high standards. In any case, autonomous vehicles still require an operational environment that facilitates the detection phases.

For these reasons, any environment where autonomous vehicles are expected to operate must establish an information exchange activity through specific devices within the ITS (Intelligent Transport System) identified as RSU (Road Side Units). Such tools communicate with AVs so that a single vehicle can be informed about a specific roadway stretch, to perform all the appropriate interventions. Moreover, communication involves the monitoring of traffic and pavement conditions, and this system is identified as SRE (Smart Road Environment) [3]. In Figure 1, it is possible to recognize an example of how 


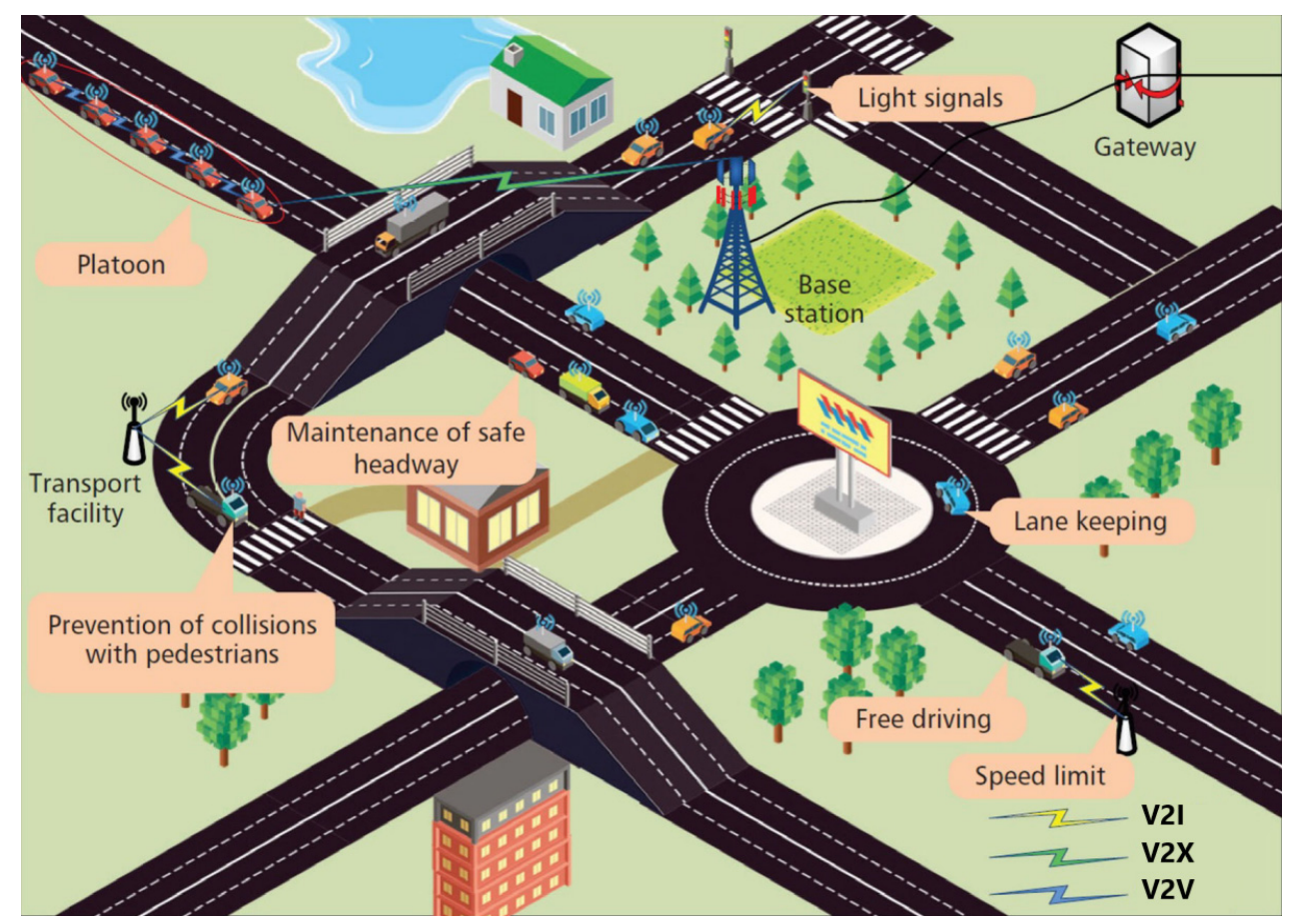

Figure 1. Example of potential SRE scenario with AVs [4].

A more accurate analysis of the advantages brought by AVs can be appreciated when making a distinction among vehicle service types; vehicles are generally classified according to their load capacity, or whether they operate for public or private service. $\mathrm{AVs}^{\prime}$ availability for private use would significantly improve urban traffic in terms of traffic management (congestion phenomena reduction) and safety, as a consequence of the high degree of precision of AVs maneuvers. Consequently, mass transit and public transport service $[5,6]$ would increase their capacity and frequency due to the possibility of reducing the headway between mass transit AVs, as they would be able to brake promptly thanks to the high number of electronic sensors. Nowadays, several AVs are already in use for public service; similarly, AVs for private use are already on the market, although mainly of level-3 type within the SAE classification. Speaking of public transport, AVs have been introduced through the adoption of electrical minibuses with a maximum capacity of 20 passengers. Their main operational scenarios are airports, parks, and hospitals, just to name a few; generally, their maximum speed can reach $25 \mathrm{~km} / \mathrm{h}$ [7]. The main real obstacle potentially slowing down the AV spread is of financial nature, as these vehicles are generally expensive for the common buyer; the minimum cost for manufacturing a single AV ranges in fact between EUR 6500 and 200,000. On the other side, AVs with their high precision performances lead to a reduction of car accidents, meaning reduced insurance fees and lower accident-related expenses [8,9].

Autonomous vehicles represent a new business in which several automotive manufacturers and transport companies are already focusing. Figure 2 shows some of the brands that have already begun investing funds in $\mathrm{AVs}$, and that are expected to strongly influence the market by 2030 [10]. 


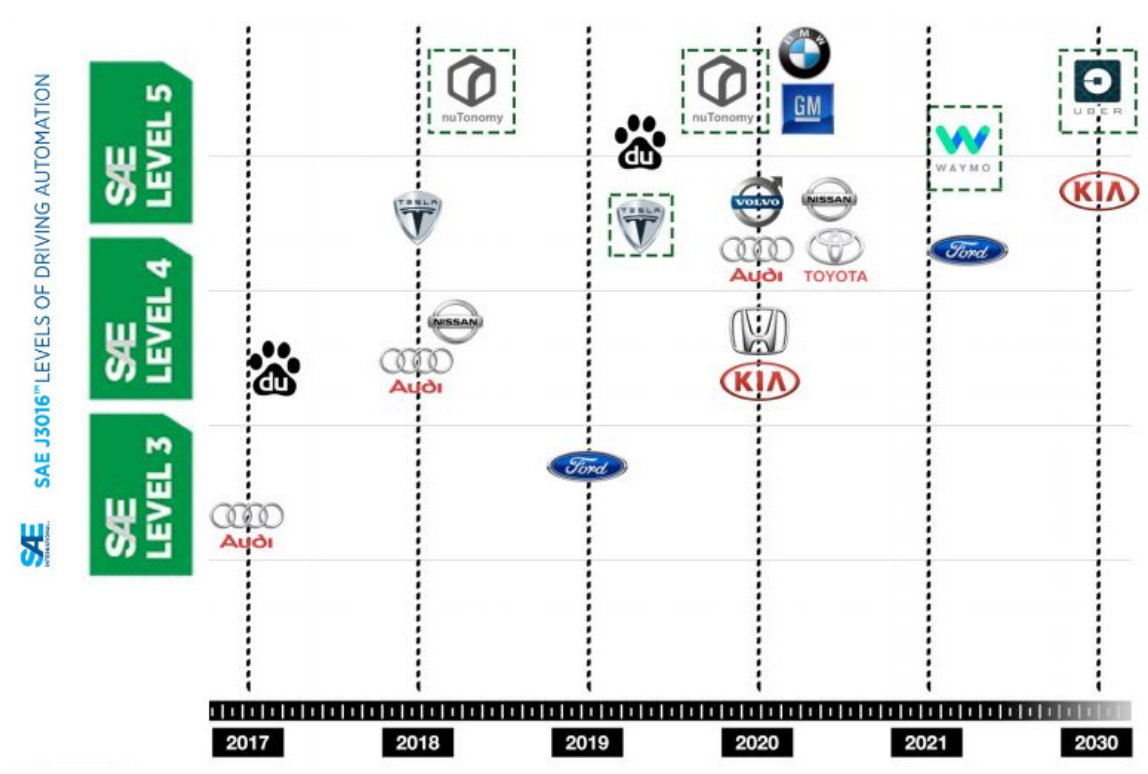

Figure 2. Evolution and expectations about automotive companies AVs market [10].

\section{State of Art: An Overview on Safety for AVs (Risks, Drawbacks, and Benefits)}

The current scientific literature on safety for AVs covers diverse technical aspects, spanning from the electronic and software progress to the interaction with other vehicles and surrounding infrastructure. The range of AV-related risks is quite broad, considering the heterogeneity of the transportation industry and how it can affect people lives in many different ways: from political decisions and governances to technology investments, from safety-related costs to user behavioral models, and from the effects on financial markets to the environmental impact, to mention a few. Through efficient risk management actions, risk reduction and mitigation should be attained in order to improve the AV technology and make it widespread to the public, primarily accepted by mass and everyday use.

Even if not strictly related to AVs' mechanical design and dynamics, a risk recently coming under the spotlight is that of health, with a particular reference to COVID-19 and by extension to other sanitary hazards. AV sharing can potentially constitute a carrier of diseases; therefore, proper measures must be identified and implemented to reduce this risk. In the case of Coronavirus, for shared and public vehicles which are used by different users, the infection can be spread mainly by contact with infected surfaces and through airborne droplets. Hence, adequate sanitation systems should be developed and implemented to allow safe sharing conditions, and to increase the sense of trust and safety perception towards shared vehicles; in fact, users might be less enticed to use shared vehicles for fear of diseases. On the other side, it is interesting to note that since AVs can be operated automatically to transport goods and carry out deliveries without the need to employ human drivers (i.e., drones) [11], the risk of transmitting diseases gets potentially reduced, as the interaction between consignees and the driver is basically absent (should they be appropriately designed to minimize contact between the various consignees); AVs are therefore useful also for delivering sanitary goods such as vaccines or bio-hazard materials during pandemics [12]. Moreover, implementing strategies and devices to monitor passengers' health status on shared AVs can help with controlling and monitoring the spread of diseases over a specific area. In this sense, there is potential for new related ideas for further research studies in the upcoming years. Figure 3 shows the Hercules logistic Autonomous Vehicle used during the COVID-19 pandemic to deliver food and medical supplies. 


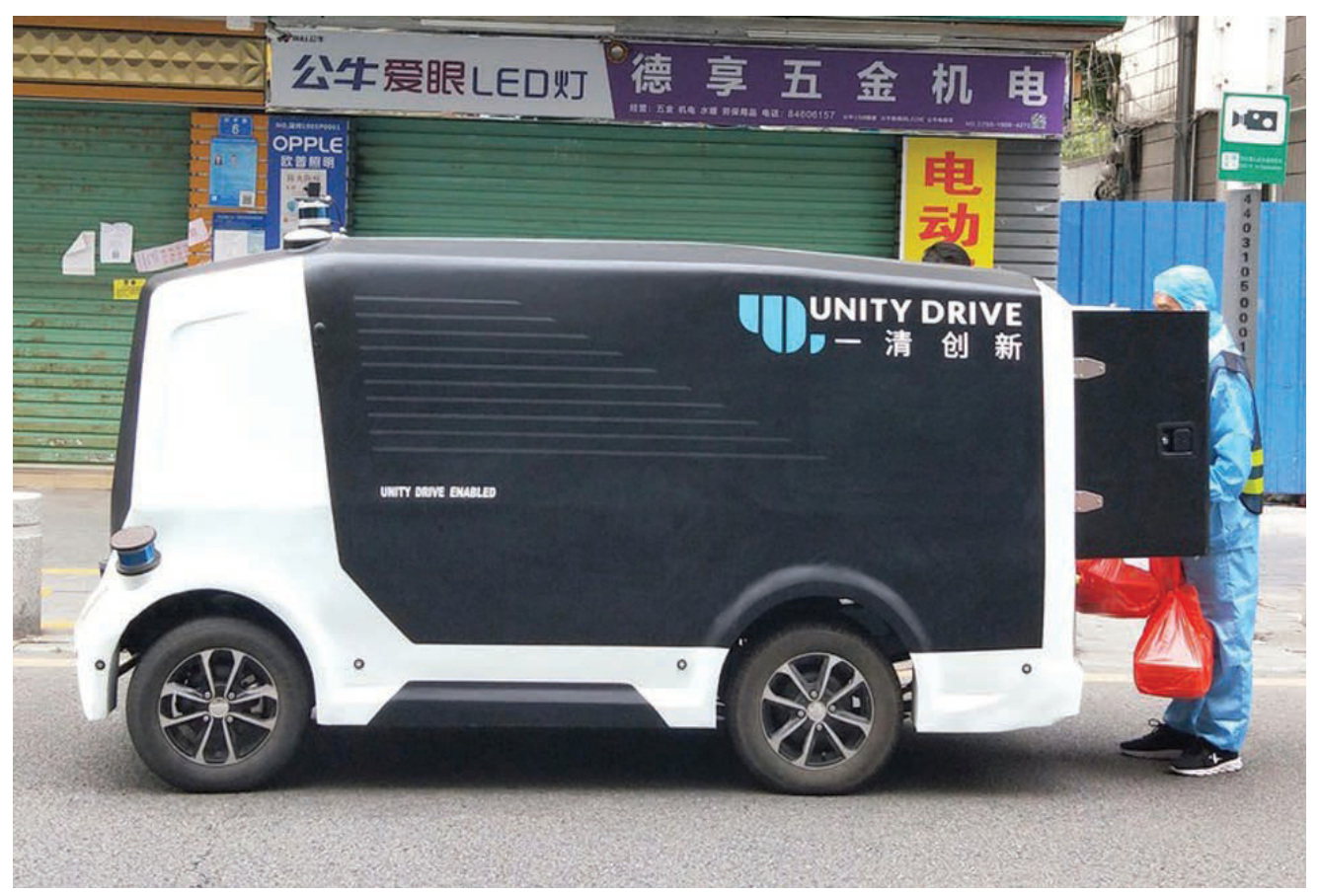

Figure 3. Hercules logistic AV used during COVID-19 pandemic to deliver goods [12].

In terms of governance, governments and agencies can adopt various strategies to manage AVs related risks depending on their visions and desired outcomes. In this type of approach, risks mainly refer to road accidents and their consequences, typically having significant impacts on people's life quality (principally physical injuries and deaths) and costs impacting national health systems and transport infrastructure. The way governments decide to intervene to manage traffic and, specifically, to regulate AV will affect how transportation systems respond in terms of safety. Examples of strategies typically vary from a no-response approach to implementing control interventions [13,14]. The adoption and spread of AVs impact the surrounding environment and territory, both urbanized and countryside, by affecting transport dynamics and user behaviors [15] such as commuting or logistics. All of these aspects reflect the way transport infrastructure is thought and built [16]. In addition, the application of AVs to public transport systems could further incentivize their use rather than smaller vehicles with lower capacity (i.e., cars) [17].

Applying these concepts to road design leads to a change in the typical paradigms of the road infrastructure design, considering, for example, all the work necessary for the implementation of those roadside electronic devices that communicate with AVs (i.e., cameras, pavement sensors, and electronic booths). All these elements must be taken into account in urban and land use planning, as they can affect parameters such as urban density, land use, impact on the environment, used materials, and emissions, to name a few. Table 1 lists some of the possible governance strategies adopted to manage AV-related risks on the transport network. 
Table 1. A brief description of several strategic approaches [17].

\begin{tabular}{|c|c|}
\hline Strategy & Description \\
\hline & $\begin{array}{l}\text { No specific actions or dedicated regulations are implemented. An } \\
\text { example might be the lack of dedicated national policies to manage }\end{array}$ \\
\hline & AVs acceptance standards, traffic operations, and related consequences. \\
\hline No-Response & Although this might seem the cheaper option, at first glance, AV-related \\
\hline & risks left alone without management can be higher in the end, leading \\
\hline & potentially to significant costs arising from the lack of risk management \\
\hline & and making it not economically convenient in the long term. \\
\hline & Risk is managed by avoidance, i.e., risk is reduced by removing the \\
\hline Prevention-oriented & $\begin{array}{l}\text { hazard, trying to prevent negative consequences by eliminating the } \\
\text { source that would cause them. An example is the prohibition of }\end{array}$ \\
\hline & AVs for certain times or areas of the road network. \\
\hline & $\begin{array}{l}\text { The hazard is here admitted and controlled by making predictions and } \\
\text { regulations as an attempt to reduce the probability that consequences }\end{array}$ \\
\hline Control-oriented & would happen. Examples of regulations could involve the mandatory \\
\hline & $\begin{array}{l}\text { requirements for AVs certified safety standards, dedicated road traffic } \\
\text { policies, use of traffic prediction models, and so on. }\end{array}$ \\
\hline & $\begin{array}{l}\text { With this approach, governments tend to make sure to be in a strong } \\
\text { and solid position when facing risks across diverse situations. An }\end{array}$ \\
\hline Toleration-oriented & example is the publication of comprehensive lists to describe \\
\hline & $\begin{array}{l}\text { contingency plans or clarify insurance liabilities while seeking } \\
\text { alternative solutions to mitigate risks. }\end{array}$ \\
\hline Adaptation-oriented & $\begin{array}{l}\text { Being an adaptation strategy, the approach consists of improving the } \\
\text { reaction caused by an existing source, accepting the hazard and its } \\
\text { uncertainties, reducing the impact of consequences, and improving } \\
\text { system performances. The aim is to achieve a resilient system. }\end{array}$ \\
\hline
\end{tabular}

Electronic and Information-Technology (IT) applied to AVs also constitute a potential risk in terms of safety. Automatic vehicles adopt electronic platforms and computer processors (Hardware, or $\mathrm{H} / \mathrm{W}$ ), along with dedicated software programs and specific code lines (Software, or S/W). Like any other technological device, these systems are subject to malfunction or failure risks, with possible consequences to the entire surrounding environment. In case of the automated systems' failure, an up-to-date backup plan managed by human beings should always be available and ready to be put into action, involving trained staff (traffic managers and constables, to name a few). In addition, additional issues may appear due to the potentially inaccurate interaction between computerized and non-computerized elements, such as pedestrians or bicycles, as the surrounding context of an AV is often unpredictable [18]. For instance, some recent studies have focused on AVs' interaction with ramp metering [19]. AV models are based on mathematical algorithms that can be translated into software [20], and dedicated studies have been developed over the last decades. Different models bear different pros and cons and should be examined singularly. Another issue concerns security, as IT systems can potentially get hacked and hijacked [21]. An adequate level of cyber-security is, therefore, necessary [22]. Since AVs work with automated systems, a well-calibrated model should guarantee precise and optimized vehicle flows, leading to improved travel times, lower traffic congestion, reduced levels of pollution, and higher safety standards. As an example, Table 2 shows how applying fitting algorithms to $\mathrm{AVs}$ ' interaction with ramp metering can lead to improved Levels of Service (LOS), lower levels of fuel consumption ( $\mathrm{g} / \mathrm{kg}$ of fuel) and emissions (grams) in the road network [23]. Acronyms reported in Table 2 are Carbon Monoxide (CO), Volatile Organic Compounds (VOC), Nitrogen Oxides (NOx), referring to the quantity of Vehicles (VEH). 
Table 2. Improvement of LOS, fuel consumption, and emissions with the application of specific algorithms [23].

\begin{tabular}{ccccccc}
\hline Scenarios & Los & Vehs & Fuel Consumption & Co & Nox & Voc \\
\hline No-Control & LOS F & 5509.00 & 3770 & 69,613 & 13,544 & 16,133 \\
ALINEA & LOS D & 5759.00 & 2319 & 42,822 & 8332 & 9925 \\
VSL & LOS D & 5692.00 & 2140 & 39,511 & 7687 & 9157 \\
VSL+ALINEA & LOS D & 5741.00 & 1902 & 35,114 & 6832 & 8138 \\
VSL+ALINEA/B & LOS C & 5904.20 & 415 & 29,024 & 5647 & 6726 \\
\hline
\end{tabular}

\section{AVs Critical Issues: Aspects Related to Specific Infrastructures Components}

The accuracy level of AV operations depends on several factors related both to vehicle tools and context characteristics. Accuracy from vehicle tools is ensured by a high number of cameras, sensors, and connections with LiDAR's systems that allow space detection during vehicle movements, while the latter depends on infrastructure characteristics, i.e., presence of ITS or RSU devices, intersection signals, and horizontal road markings [24]. When considering high-performance detection systems for AVs maneuvers, roadway geometric layouts do not require significant upgrades, whereas pavements might require more invasive interventions. Pavements are made of several layers characterized by constant thickness across each horizontal cross-section, an aspect to be taken into account when upgrading the road infrastructure due to correlation with the AVs maneuvers causing a reduction of the "vehicle wandering" phenomenon.

\subsection{Autonomous Vehicles Detection Capability at Intersection and for Horizontal Road Markings}

The quality of horizontal road markings plays a crucial role for autonomous vehicle detection, not only to avoid conflicts but also to facilitate pedestrian individuation, for instance, in zebra crossing points. Hence, ADAS (Advanced Driving Assistance Systems) equipped by $\mathrm{AVs}$ must ensure an efficient and smart detection of road markings, primarily due to their frequent deterioration (mainly caused by severe weather and actions from rolling wheels) and also to poor maintenance. Road markings are fundamental for vehicle lane-keeping, with straight or curved lines substantially characterizing these signal types; additionally, horizontal markings may also include directional arrows, helpful for the $\mathrm{AVs}^{\prime}$ decision-making process. However, directional arrows can be perceived differently than simple lines by visual cameras due to several factors like angles of view, line thickness or interference of shadows projected by nearby objects. In AVs, the detection process of horizontal road markings occurs through vision-based systems that result to be the more appropriate, mainly for economic reasons thanks to the inexpensiveness of vision cameras. Moreover, these systems also allow the creation of 3D input data whose quality is optimal as one of LiDAR's systems [25], useful considering that visual data is the most suitable input for human drivers.

Therefore, road marking detection has a dual approach, the first related to AV detection capabilities and the second to the quality of horizontal road markings. The latter represents an aspect of vehicle circulation affecting safety regardless of autonomous performances and MV (machine vision) capabilities. Since 1992, Miller highlighted how to correct maintenance of road markings involves at least sixty times less than costs caused by incidents caused by a lousy perception of roadway spaces. The parameter that mainly affects MV's road markings perception is the retro-reflectivity (RL), especially in low light hours. Such feature represents the capability to reflect radiations and can be measured as $\mathrm{mcd} / \mathrm{m}^{2} / \mathrm{lx}$. In 2016, Matowicki et al. demonstrated that, when road markings had an $\mathrm{RL}=50 \mathrm{mcd} / \mathrm{m}^{2} / \mathrm{lx}, \mathrm{MV}$ systems began to show malfunctions, while other studies, like the one of Davies in 2017, reported that MV was also affected by width and color, where white was more suitable to be detected compared to yellow [2].

Several methods have been studied to facilitate road markings detection with various processes. For example, Hechri and Adellatif provided a system characterized by two video-based driving tasks. At the same time, Lee combined AVM camera features with 
LiDAR's data. Ultimately, Zhen Kang and Qiao Zhang proposed an algorithm able to speed up map rendering by solving point cloud data [26]. Some of these studies were also analyzed through a forecasting process with simulation software. Le-Anh Tran and My-Ha Le carried out their proposal of a robust road lane markings detection system with simulator CARLA that showed how the system resulted inefficient in straight parts. At the same time, it did not present beneficial results in curves [27].

It is possible to state that the quantity of horizontal markings is related to the roadways context dynamism, meaning that, whenever the possibility of change directions and actors being part of a specific context is elevated, the high quantity of roadway markings will characterize the environment. Therefore, intersections represent a critical issue for marking detection, but for instance, a vital detection challenge for AV sensors is the work area. Work zones are subject to unexpected continuous variations, mainly due to vehicle movement. That is why horizontal markings have a crucial role in implementing a safe work context, especially in highways or freeways where speed ranges are elevated. For these reasons, some studies considered proposing new pavement markings for work zones, as reported in Table 3 and, in some cases, with no predetermined itinerary vehicle so with driver presence. Generally, in a work zone, a significant trial for autonomous vehicles is the reduced lane width between 3.5 and $2 \mathrm{~m}$, if not even less. Different types of barriers or furniture can also be positioned in such lanes that the AV detected as obstacles can cause vehicle sudden stops when is not required. Ultimately, one last critical aspect consists of using various pavement marking materials to cause a high quantity of data collection for $\mathrm{AVs}$ when required just the same function or markings detection. Then it would be helpful to the deployment of uniform materials [28]. One aspect that can help solve the aforementioned problems is the lower speed in work zones that allow detection sensors to get better environment perceptions. Therefore, speed range is fundamental [29]. Highways or freeways can be subjected to many conflicts if signals and markings are not efficiently detectable. It has to be highlighted that autonomous vehicles' processes related to environment detection can co-occur to a data management and decision-making process of multiple vehicles handled by a central infrastructural system. Then, it is helpful to apply methods that facilitate the operation previously cited. For example, it is possible to apply protocols related to vehicle intersections behavior based on V2V communication. Some of these protocols are Advanced Maximum Progression Intersection Protocol (AMP-IP) and Maximum Progression Intersection Protocol (MP-IP). MP-IP objective is intersection throughput increase concerning safe crossings until its primary goal of passage safety is satisfied all vehicles are allowed to cross the intersection, also in case of potential conflicts. AMP-IP considers a general conflict cell, allows low-priority cars to cross the intersection before high-priority vehicles arrive [30]. Automated Intersection Management or Intersection Managers (IM) are systems designed to interact with vehicles approaching intersections, aiming to improve traffic safety and efficiency.

One of these systems' benefits is that they can reduce the probability of accidents caused by human mistakes, often due to improper driving evaluations or impulsive driving behaviors, due to emotional decisions or instinctive actions rather than logical analysis [31]. Driving reaction times during emergency maneuvers are very short and often will not leave the driver enough time to decide the next move wisely. Automated Intersection Management (IM) systems work by exploiting the communication between vehicles and devices placed on intersections. Vehicles approaching the intersection at constant speed transmit a speed query to the IM, receiving a Yes/No reply depending on other simultaneous requests from other vehicles to optimize flow and manage safety. In case of a positive reply, the incoming vehicle is authorized to proceed and can drive forward, crossing the intersection at the requested constant speed. Should the answer be negative, in that case, the vehicle will slow down and resend new queries to the IM. Once the vehicle crosses the intersection, another signal is sent to the receiving device, communicating that the maneuver has been completed. This system increases the admissible road traffic volume and relies on a large and increasing amount of data to be exchanged between AVs and IM. Hence, its efficiency 
depends on the accuracy of this shared data. Robust Intersection Manager's system adds the benefit of tracking vehicles' trajectories approaching an intersection associated with a Velocity of Arrival (VOA) and Trajectory of Arrival (TOA) as they get close to the junction. This situation leads to increased accuracy and higher safety levels. This system is still subject to hardware or communication errors and even to hacking attempts interfering with vehicle positioning and speed detection. Figure 4 below depicts the dynamics of an accident happening with these systems.

Table 3. New markings for AVs movement in work areas [28].

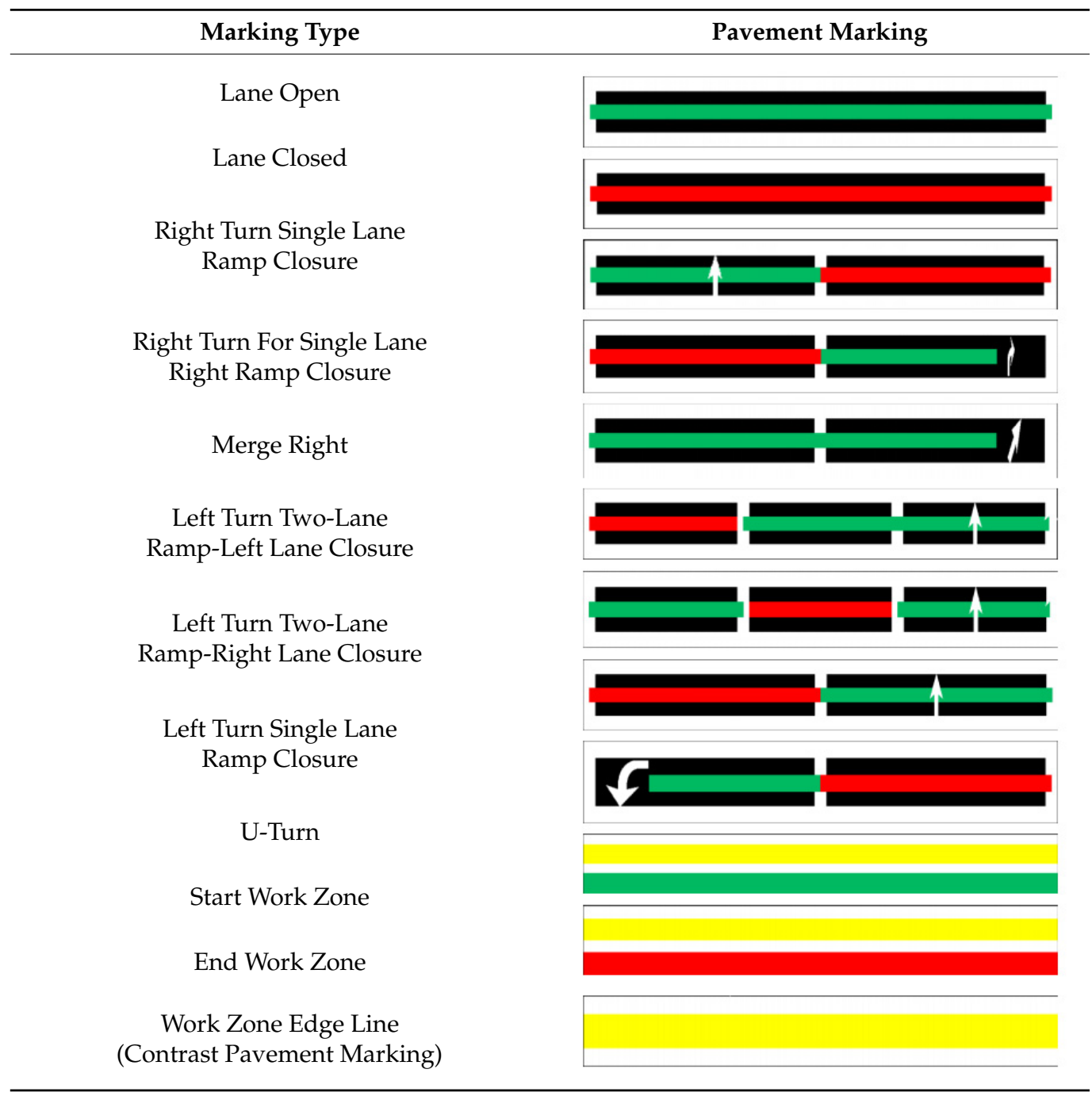

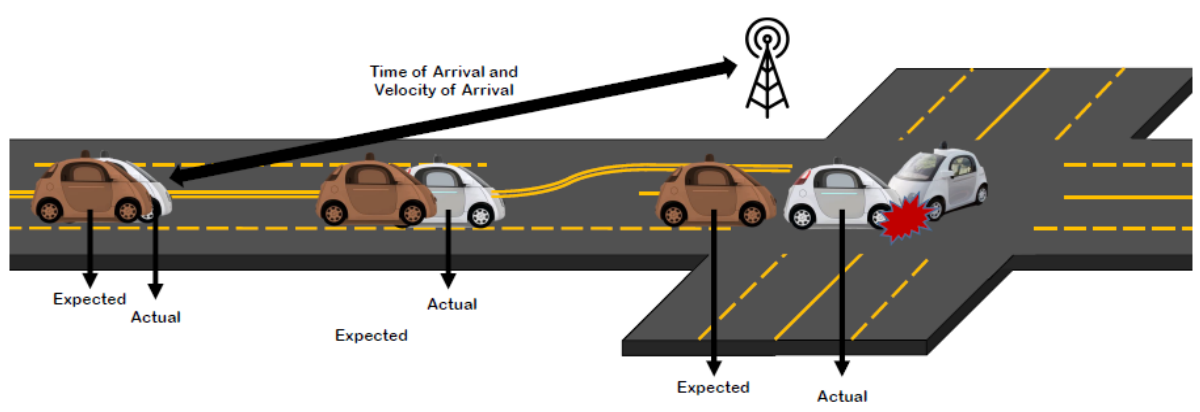

Figure 4. Accident dynamics on IM systems [31]. 
The use of new strategies, based, for instance, on the Right-of-Way Assignment (RWA), can improve traffic dynamics in the presence of non-signalized intersections. This strategy is based on marking short-term decisions for the right of ways and on one-to-one communications. Other assumptions include that two vehicles can cross the intersection simultaneously and that pilot vehicles can obtain information about speed, location, and driving intentions of other surrounding vehicles. Moreover, priority levels and precedence rules are defined. A related strategy, called Cooperative Driving Strategy (CDS), introduces the assumption and possibility that the information connecting all the elements involved in traffic (pedestrians, bicycles, and vehicles, to name a few) is available via a V2X communication system, which can cover up an area of $300 \mathrm{~m}$ or even more. This methodology splits the junction in a grid made of several smaller areas, regulating vehicle traffic by setting up the minimum time interval between two vehicles. Results from dedicated research studies [32] show that this approach leads to higher performances compared, for example, to a traditional Responsibility Sensitive Safety (RSS) approach (which applies a typical First-In/First-Out (FIFO) strategy with few communications). Figures 5 and 6 show a significant difference in performances between RWA/CDS and RSS, specifically in terms of average delay/arrival date and throughput/arrival rate ratios.

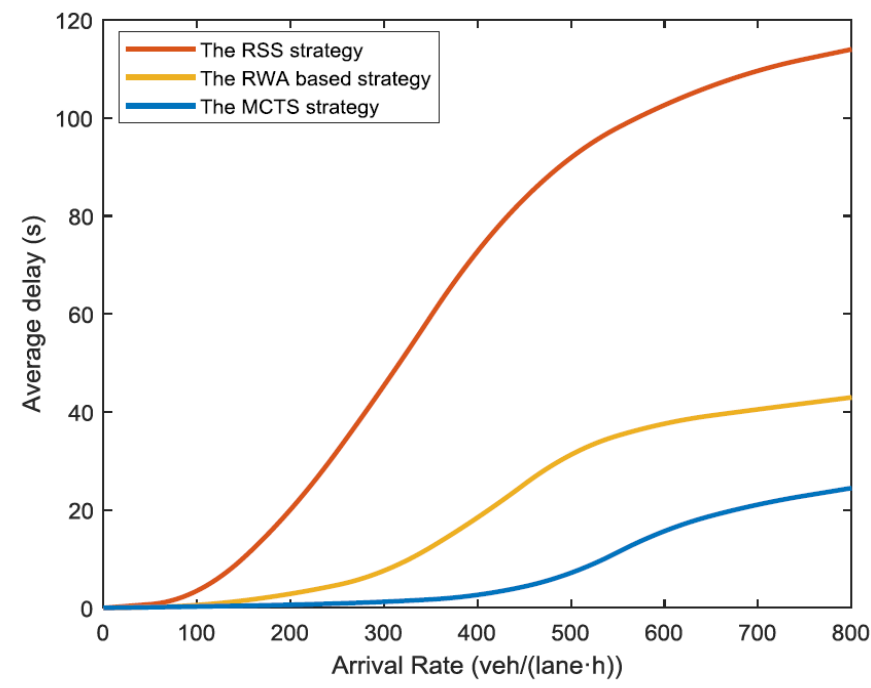

Figure 5. Comparison between RWA, CDS, and RSS in terms of average delay/arrival rate [32].

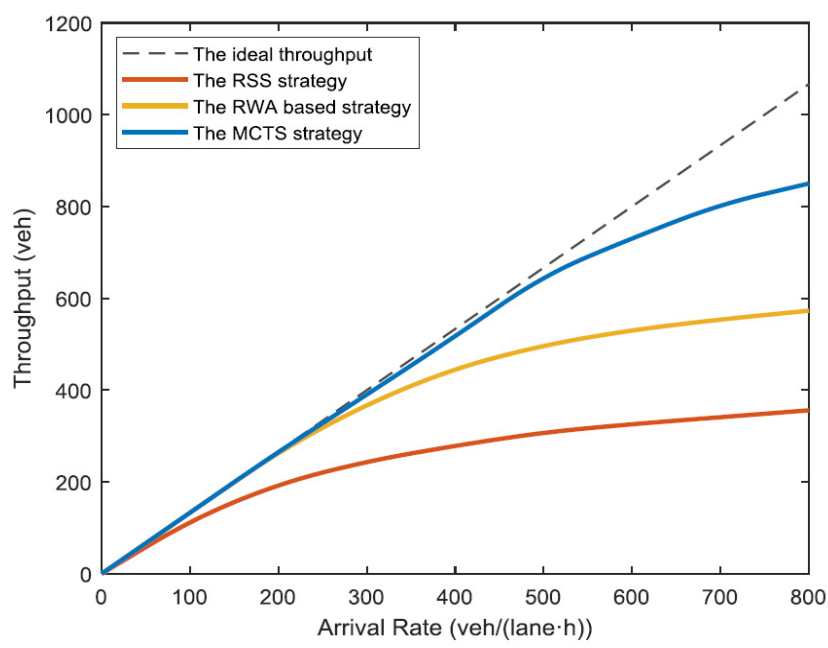

Figure 6. Comparison between RWA, CDS, and RSS in terms of throughput/arrival rate [32]. 
Simulation models for AVs at intersections can also be trained thanks to machine learning, specifically Reinforcement Learning (RL) and computer vision. Aerial photographs can provide visual information about the road intersection to create a simulated model. The vehicles' position, both autonomous and manned, can be detected using deep neural networks of various kinds [33]. Vehicles in the intersection can be detected with the segmentation technique, starting from a full-color aerial image of the crossroad captured with drones and then post-processed, moving through computerized Fully Convolutional Network (FCN), creating a B/W image which then becomes clustered through a DBSCAN. With the OpenCV CVAT software tool, vehicles on the aerial images are marked and labeled with polygons (rectangles and ellipses), indicating the relative location. Possible trajectories are marked with polylines as well. Vehicles are finally manually removed from the image, leaving the polygons on. The image becomes then binarized and clustered into closed regions with the DBSCAN algorithm, filtering and discarding the smaller regions. From this point onwards, the simulation is undertaken thanks to the application of complex neural network algorithms that eventually lead to a calibrated and portable model, which can then be included in larger Automated Intersections systems.

Like RL and computer vision, Neural networks Systems (and generally speaking systems based on machine learning) often require advanced and expensive hardware tools like for example survey drones with cameras and powerful computers, which are not always affordable for common application. However, they can be seen as an example of how current technology for AVs has become sophisticated, considering how drones and surveying sensors have quickly become mainstream in recent years. In addition, there is always the risk of malfunctions or even hacking attempts to cause some damage. In addition to technical constraints, which can affect accuracy and influence costs, the use of drones might require special authorizations and licenses, which is another parameter to consider. Negligence, in this sense, might lead, for example, to fines, trials, or any other legal inconvenience with reputational and financial consequences. On the other hand, the achieved benefits are related to traffic optimization and its subsequent effects, the portability and flexibility of the simulation models, and the improvement of the modeling accuracy. The flowchart in Figure 7 summarizes the process adopted in this method.

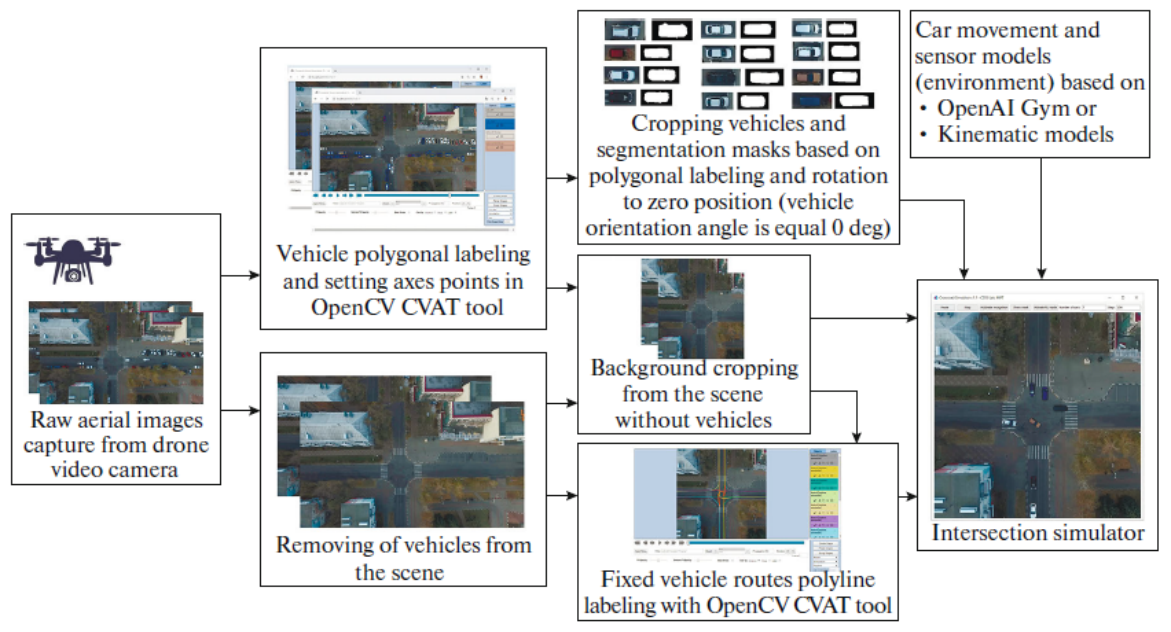

Figure 7. Flowchart of the RL: computer vision process for an intersection [33].

It is helpful to remind, with a brief excursus, that artificial or electronic neural networks are computational systems inspired by and resemble biological neural networks, i.e., the complex system of neurons (nodes) and synapses (connectors) typical of animal brains. These systems allow the reconstruction of real-life scenarios characterized by the presence of scattered and interacting elements. It is beneficial, for instance, in camera detection whenever the nodes are hard to spot due to bad weather conditions, low light conditions, or obstacles concealing them [34]. Image segmentation can be performed with systems 
like Structured Inference networks [35], based on the assumption that the analyzed data conforms to a Gaussian State Space Model (GSSM). Commonly, algorithms to train neural networks fall into three major types: Levenberg-Marquardt, Bayesian Regularization, and Scaled Conjugate Gradient [36]; adopting the best fitting algorithm depends indeed on the geometry of the collected dataset and the surrounding scenario. Deepening into Artificial Intelligence and Machine Learning would require a reliable dissertation as the subject is pervasive and has its particular field of study. Therefore, it will not be treated in detail in this article. Other similar approaches allow modeling vehicle trajectories and their variations for vehicles turning at intersections [37]. One of the methodologies adopted for this purpose exploits the principle of "Minimum Jerk", i.e., a model initially developed to describe a human arm's biodynamics in a plane, later extended to robotics eventually to AVs for studies on motion control. This model states that a jerk function can represent movements performed by the arm to carry out tasks like writing or drawing. The minimum jerk is found by minimizing this function through integration within an interval between a given initial location and a final location within a given time. The Minimum Jerk model and other support models aimed to identify minimum speeds and the vehicle-related position [38,39]. When inserted into a simulated framework, these allow estimating turning vehicles' trajectories at an intersection. These estimations can be incorporated into automatic intersections to achieve the benefits already mentioned in this paper. It is interesting to remind that simulations created with all these models must also deal with human driving behaviors, which statistically depends on parameters [40], such as age, driving skills and training, and state of lucidity and attention, not to mention all those random parameters that could be present (even the presence of an insect could disturb the driver and cause an accident). The implementation of AVs [41] would ideally fix these issues, as the model's random or less predictable components are removed since the driver is absent. This situation should lead to even higher optimization levels, increasing safety and reducing travel times, to mention a couple of benefits. Figure 8 summarizes with a flowchart the process and steps that define the Minimum Jerk approach. In contrast, Figure 9 shows an example of the computer output, reporting the comparison between paths (a), speed profiles (b), acceleration profiles (c), aggregated speed profiles (d), and aggregated acceleration profiles (e) for different exit speeds.

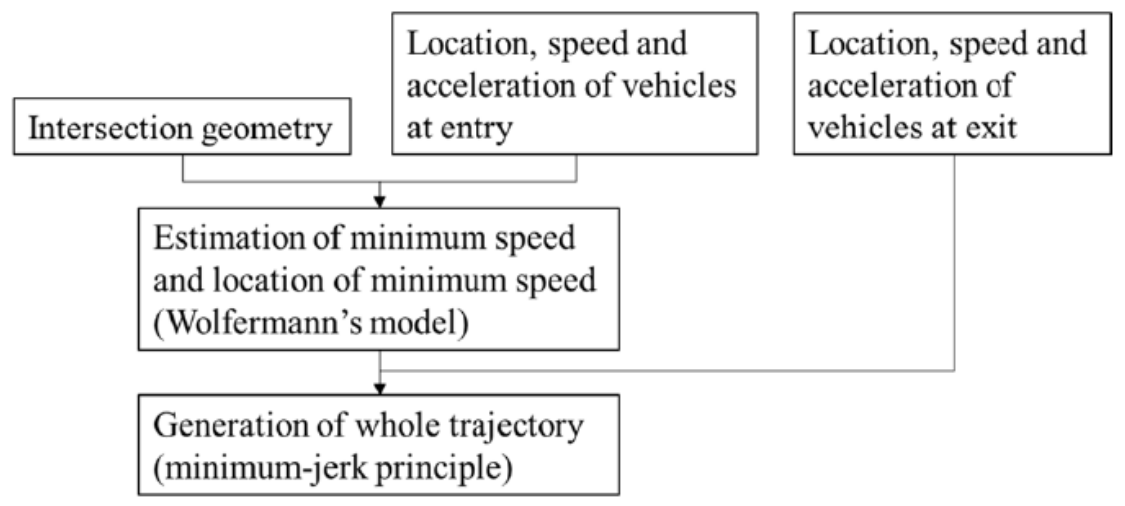

Figure 8. Flowchart of the process for Minimum Jerk model [37]. 

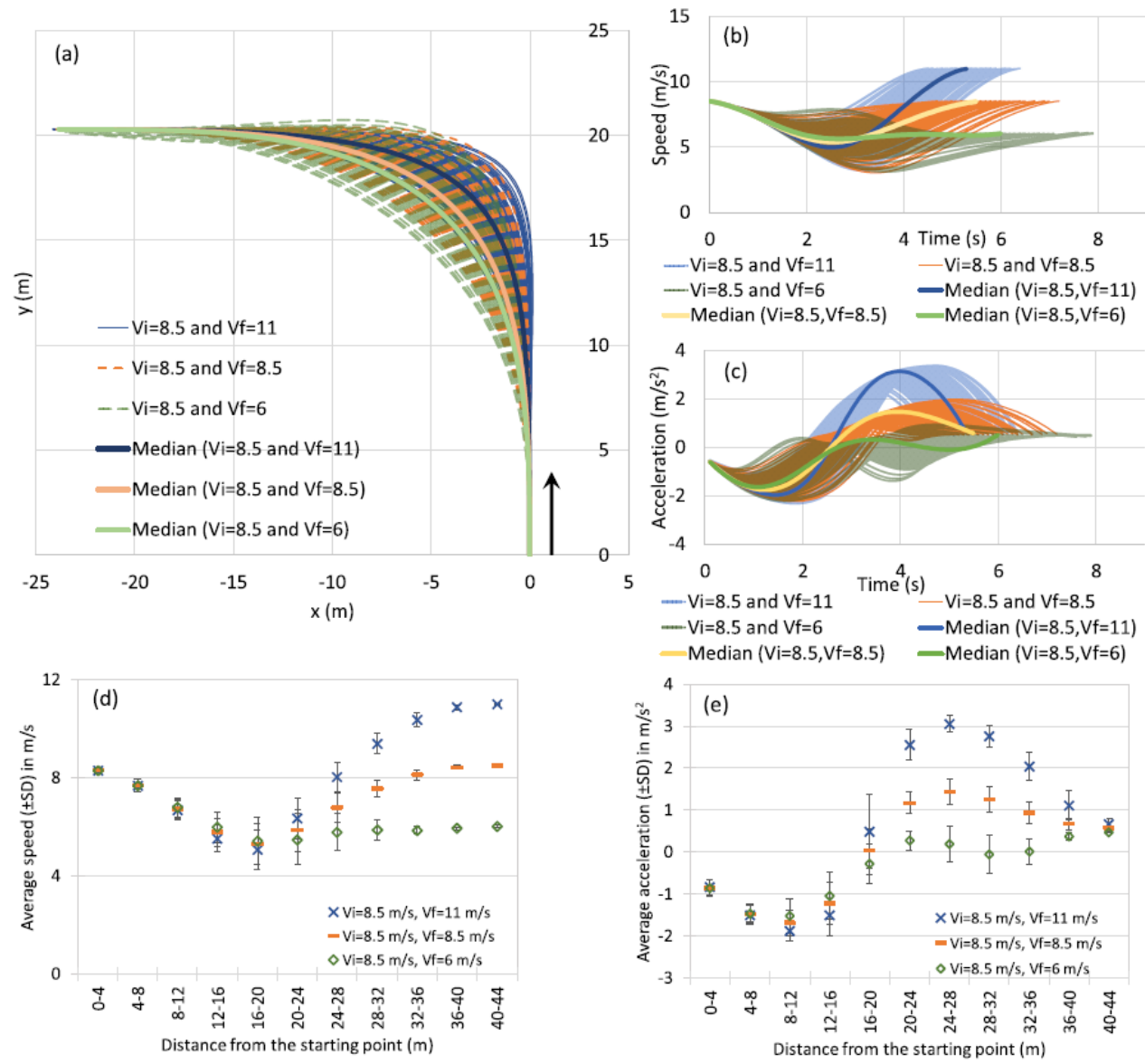

Figure 9. Computer output from a Minimum Jerk model—comparison between several traffic parameters for various exit speeds [37].

\subsection{AVs Effects on Roadway Pavement}

Once treated themes related to $\mathrm{AVs}^{\prime}$ advantages and expected consequences, it is necessary to focus on infrastructural design features [42,43]. One aspect can regard the change of load layout applied on road pavement. As previously argued, AVs would be able to keep with high precision in lane position. It is possible to consider that each vehicle's wheels would stress road pavement always in the same areas and with almost constant speed in a context aimed at AVs exclusive transition. Ultimately, it is expected that road pavement would be stressed by vehicle loads not homogeneously, like for vehicles driven by a human driver (since each driver has its way of driving), but in a cross-section area that could be almost constant [44,45]. Table 4 reports an overview of AVs' effects on roadway pavement.

Table 4. Overview of AVs effects on pavement [46].

\begin{tabular}{cl}
\hline Effect & \multicolumn{1}{c}{ Consequence } \\
\hline Channelized traffic flow & $\begin{array}{l}\text { Easier forecasting process related to cracking and } \\
\text { deformation detection. } \\
\text { Pavement surface optimization due to higher frequency } \\
\text { of load application. }\end{array}$ \\
$\begin{array}{c}\text { Speed load frequency } \\
\text { Acceleration/deceleration } \\
\text { optimization }\end{array}$ & Reduction of longitudinal pavement surface stress. \\
$\begin{array}{c}\text { Stop/start maneuvers reduction } \\
\text { at intersection }\end{array}$ & Incidents reduction, traffic management facilitations. \\
Traffic increase at night & Increased pavement life-cycle due to lower temperature. \\
\hline
\end{tabular}


With the introduction of autonomous vehicles, ordinary traffic will be less subjected to vehicles' wandering phenomena than channelized traffic. Such conversion can have several consequences, such as greater rutting, significantly increasing longitudinal fatigue cracks. In this case, heavy vehicles would have to be mainly analyzed because tires could cause an elastic deflection bowl. This phenomenon is attributed to the possibility that AVs provide to reduce heavy vehicle headways. It was demonstrated by De Beer (1992) that, considering loads cycle application applied quicker, due to high frequency related to headway reduction, road pavement keep a slightly deflected condition that is, in turn, stressed again by following load axis application.

So, the subsequent deflection will be higher. Such issues can be managed with a technique named "strip road concept," consisting of installing two parallel strips made of asphalt, concrete, or improved soil positioned in correspondence to specific vehicles' wheels that will transit on that roadway. Significant localized stress pavement is subjected to AVs. It was detected during accelerated pavement testing (APT). It is noted, in Figure 10, different pavement responses under expected $\mathrm{AVs}$ and conventional vehicle loading respectively channelized and wandering traffic conditions standard hot mix asphalt surfaced.

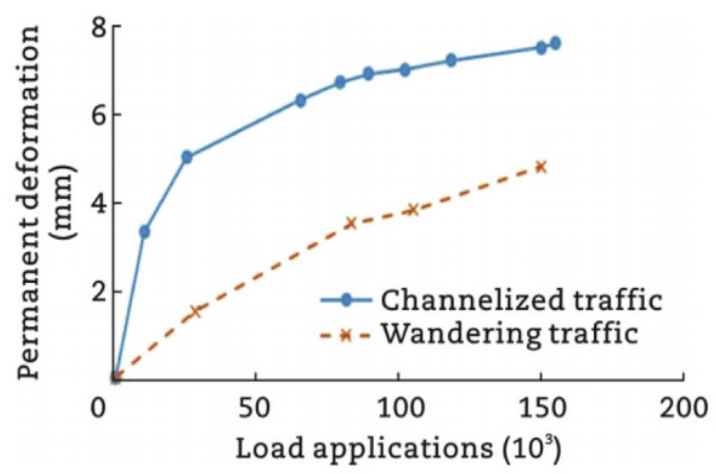

Figure 10. Channelized versus wandering permanent deformation response [46].

\section{Discussion}

AVs are a relatively recent technology, developed and spread over the last decades and now becoming more common in daily life, thanks to technology's progress, particularly in the IT field. The most recent AV technology exploits mathematical algorithms converted into software codes to regulate automated traffic and maneuvers undertaken at road intersections [47]. Neural networks have been implemented for this purpose too. One of the main challenges, in this sense, consists of the accuracy levels achieved when replicating the analysis scenario, as it can be characterized by a multitude of different variables that cannot always be translated into computerized models (for instance, trying to predict pedestrian traffic trajectories). Accuracy also depends on the type of algorithm used for the scenario. The best results are achieved the more the algorithm fits the objective case. Calibration plays another critical role in this sense. General considerations for some of the AV algorithms have been introduced in this article, explicitly concerning automated intersections. AVs technology offers ongoing opportunities for upgrades and improvements. Therefore, the goal to replicate real-life models and random interactions with decent accuracy still constitutes a significant challenge for multidisciplinary research studies. Regarding road intersections, one of the most common approaches considers the interaction between vehicles, both autonomous and unmanned, and special devices installed at the intersection to detect and interact with traffic, optimizing velocities and space between vehicles in order to achieve the best and most certain traffic conditions.

AVs bring many benefits to several areas, considering the improvement in road safety, reduction of emissions, and travel time optimization, to name a few. Additional benefits concern health as these vehicles deliver sensitive goods without exposing any driver to them (for instance, delivering hazardous substances to hospitals). Current scientific 
literature already shows the benefits introduced by AVs, improving day by day research progresses with its course. An optimized traffic flow means reducing travel times and lower congestion levels, consequently reducing pollution emissions. Moreover, since maneuvers become automatized, the probability of accidents caused by human errors and misbehavior becomes lower, with clear consequences for people's quality of life and government services costs. Although reducing some risks, AVs introduce new ones, some of which have been reported in this article, risks related to errors and malfunctioning of the automated systems, and consequent to hacking attempts. As a proposed idea, it could be interesting, for example, to make a comparative study between some of the reduced risks against the introduced ones, especially trying to assess and compare the impact of possible consequences. One of the most significant challenges would probably lie in their assessment and attempt to translate them into comparable numerical values (safety, direct and indirect costs, and emissions, to name a few) [48]. The research could also focus on improving the existing algorithms, analyzing new scenarios, deepening recent aspects such as the COVID19 and its effects on traffic automation. In any case, it is understandable how the research in AVs will be helpful in the following years and will have to proceed side by side with several different disciplines such as IT, electronics, and even medicine.

\section{Conclusions}

The main objective pursued by this work is to stimulate interest in Autonomous Vehicles, presenting some of the benefits that can be achieved with their implementation within road networks. Through a review of the most recent dedicated literature, this manuscript firstly introduces the basics of AVs, describing their background and evolution through time, mentioning some of the main uses (for example, the delivery of bio-hazardous substances during pandemics), what are the possible approaches adopted by governments to regulate $\mathrm{AVs}$, and some among $\mathrm{AV}$ related risks. The interaction of $\mathrm{AVs}$ with automated intersections is also examined, highlighting the technical risks that arise from implementing these systems and mentioning some of the models adopted for simulations. Eventually, the impact of AV on road pavements is also assessed. Future research can focus on many AV aspects, especially regarding security and environmental impacts, considering how new risks arise as technology advances (Artificial Intelligence, new electronic sensors, etc.), together with the improvement of computer alghorithms and new studies on behavioral models.

Author Contributions: The authors contributed equally to this work. All authors have read and agreed to the published version of the manuscript.

Funding: This research received no external funding.

Institutional Review Board Statement: Not applicable.

Informed Consent Statement: Not applicable.

Acknowledgments: This work was related to the D.D. 407 of 27 February 2018 AIM-Attrazionee MobilitàInternazionale, issued by the Italian Ministry of Education, University, and Researchin implementation of Action I.2 Mobilitàdei Ricercatori Asse I-PON R\&I 2014-2020, taking intoaccount the written amendment procedure of the PON R\&I 2014-2020, pursuant to articles 30 and 90 of Regulation (EU) 1303/2013 started on 21 February 2018, as well as the relevant implementationregulations.

Conflicts of Interest: The authors declare no conflict of interests.

\section{References}

1. Arvin, R.; Khattak, A.J.; Kamrani, M.; Rio-Torres, J. Safety evaluation of connected and automated vehicles in mixed traffic with conventional vehicles at intersections. J. Intell. Transp. Syst. 2020, 25, 170-187. [CrossRef]

2. Burghardt, T.E.; Mosböck, H.; Pashkevich, A.; Fiolić, M. Horizontal road markings for human and machine vision. Transp. Res. Procedia 2019, 48, 3622-3633. [CrossRef]

3. Trubia, S.; Severino, A.; Curto, S.; Arena, F.; Pau, G. Smart Roads: An Overview of What Future Mobility Will Look Like. Infrastructures 2020, 5, 107. [CrossRef] 
4. Bagloee, S.A.; Tavana, M.; Asadi, M.; Oliver, T. Autonomous vehicles: Challenges, opportunities, and future implications for transportation policies. J. Mod. Transp. 2016, 24, 284-303. [CrossRef]

5. Susanna, A.; Crispino, M.; Giustozzi, F.; Toraldo, E. Deterioration trends of asphalt pavement friction and roughness from medium-term surveys on major Italian roads. Int. J. Pavement Res. Technol. 2017, 10, 421-433. [CrossRef]

6. Świderski, A.; Jóżwiak, A.; Jachimowski, R. Operational quality measures of vehicles applied for the transport services evaluation using artificial neural networks. Ekspolatacja Niezawodn. Maint. Reliab. 2018, 20, 292-299. [CrossRef]

7. Trubia, S.; Severino, A.; Curto, S.; Arena, F.; Pau, G. On BRT Spread around the World: Analysis of Some Particular Cities. Infrastructures 2020, 5, 88. [CrossRef]

8. Duarte, F.; Ratti, C. The Impact of Autonomous Vehicles on Cities: A Review. J. Urban Technol. 2018, 25, 3-18. [CrossRef]

9. Bösch, P.M.; Becker, F.; Becker, H.; Axhausen, K.W. Cost-based analysis of autonomous mobility services. Transp. Policy 2017, 64, 76-91. [CrossRef]

10. Favarò, F.M.; Nader, N.; Eurich, S.O.; Tripp, M.; Varadaraju, N. Examining accident reports involving autonomous vehicles in California. PLOS ONE 2017, 12, e0184952. [CrossRef]

11. Tasiguano, C.; Danny, Z.; Alex, T.; Camacho, O.; Alvaro, P.; Ananganó Alvarado, G. A review of autonomous vehicle technology and its use for the COVID-19 contingency. In Artículo de Investigación. Revista Ciencia e Ingeniería; Universidad de los Andes (ULA): Merida, Venezuela, 2021; Volume 42, pp. 43-52, ISSN 1316-7081; ISSN Elect. 2244-8780.

12. Liu, T.; Liao, Q.H.; Gan, L.; Ma, F.; Cheng, J.; Xie, X.; Wang, Z.; Chen, Y.; Zhu, Y.; Zhang, S.; et al. The Role of the Hercules Autonomous Vehicle during the COVID-19 Pandemic: An Autonomous Logistic Vehicle for Contactless Goods Transportation. IEEE Robot. Autom. Mag. 2021, 28, 48-58. [CrossRef]

13. Li, Y.; Taeihagh, A.; De Jong, M. The Governance of Risks in Ridesharing: A Revelatory Case from Singapore. Energies 2018, 11, 1277. [CrossRef]

14. Taeihagh, A.; Lim, H.S.M. Governing autonomous vehicles: Emerging responses for safety, liability, privacy, cybersecurity, and industry risks. Transp. Rev. 2018, 39, 103-128. [CrossRef]

15. Sun, S.; Wong, Y.D.; Liu, X.; Rau, A. Exploration of an integrated automated public transportation system. Transp. Res. Interdiscip. Perspect. 2020, 8, 100275. [CrossRef]

16. Mouratidis, K.; Peters, S.; van Wee, B. Transportation technologies, sharing economy, and teleactivities: Implications for built environmentand travel. Transp. Res. Part D Transp. Environ. 2021, 92, 1027. [CrossRef]

17. Ceder, A. Urban mobility and public transport: Future perspectives and review. Int. J. Urban Sci. 2020, 1-25. [CrossRef]

18. Riccardo Mariani, R. An Overview of Autonomous Vehicles Safety. In Proceedings of the 2018 IEEE International Reliability Physics Symposium (IRPS), Burlingame, CA, USA, 11-15 March 2018; pp. 6A.1-1-6A.1-6. [CrossRef]

19. Trubia, S.; Curto, S.; Barberi, S.; Severino, A.; Arena, F.; Pau, G. Analysis and Evaluation of Ramp Metering: From Historical Evolution to the Application of New Algorithms and Engineering Principles. Sustainability 2021, 13, 850. [CrossRef]

20. Arjun, P. Comparative Study of Artificial Intelligence Algorithms for Autonomous Vehicle. Int. J. Sci. Res. (IJSR) 2020, 9, 1579-1584. Available online: https:/ / www.ijsr.net/search_index_results_paperid.php?id=ART20204360 (accessed on 16 April 2021).

21. Khan, S.K.; Shiwakoti, N.; Stasinopoulos, P.; Chen, Y. Cyber-attacks in the next-generation cars, mitigation techniques, anticipated readiness and future directions. Accid. Anal. Prev. 2020, 148, 105837. [CrossRef]

22. Chowdhury, M.; Islam, M.; Khan, Z. 'Security of Connected and Automated Vehicles', The Bridge. Natl. Acad. Eng. 2019, 49, 46-56.

23. Dadashzadeh, N.; Ergun, M. An Integrated Variable Speed Limit and ALINEA Ramp Metering Model in the Presence of High Bus Volume. Sustainability 2019, 11, 6326. [CrossRef]

24. Zhao, J.; Xu, H.; Liu, H.; Wu, J.; Zheng, Y.; Wu, D. Detection and tracking of pedestrians and vehicles using roadside LiDAR sensors. Transp. Res. Part C Emerg. Technol. 2019, 100, 68-87. [CrossRef]

25. Hoang, T.M.; Nam, S.H.; Park, K.R. Enhanced Detection and Recognition of Road Markings Based on Adaptive Region of Interest and Deep Learning. IEEE Access 2019, 7, 109817-109832. [CrossRef]

26. Kang, Z.; Zhang, Q. Semi-automatic road lane marking detection based on point-cloud data for mapping. J. Phys. Conf. Ser. 2020, 1453. [CrossRef]

27. Tran, L.A.; Le, M.H. Robust U-Net-based Road Lane Markings Detection for Autonomous Driving. In Proceedings of the 2019 International Conference on System Science and Engineering (ICSSE), Dong Hoi, Vietnam, 20-21 July 2019.

28. Singh, P.; Islam, M.A. Movement of Autonomous Vehicles in Work Zone Using New Pavement Marking: A New Approach. J. Transp. Technol. 2020, 10, 183-197. [CrossRef]

29. Choi, M.; Rubenecia, A.; Choi, H.H. Reservation-Based Intersection Crossing Scheme for Autonomous Vehicles Traveling in a Speed Range. In Proceedings of the 2019 International Conference on Information Networking (ICOIN), Kuala Lumpur, Malaysia, 9-11 January 2019.

30. Chuprov, S.; Viksnin, I.; Kim, I.; Nedosekin, G. Optimization of Autonomous Vehicles Movement in Urban Intersection Management System. In Proceedings of the 24th Conference of Open Innovations Association (FRUCT), Moscow, Russia, 8-12 April 2019.

31. Dedinsky, R.; Khayatian, M.; Mehrabian, M.; Shrivastava, A. A Dependable Detection Mechanism for Intersection Management of Connected 2 Autonomous Vehicles Aviral Shrivastava. In Workshop on Autonomous Systems Design (ASD 2019); Schloss Dagstuhl-Leibniz-Zentrum fuer Informatik: Wadern, Germany, 2019. [CrossRef] 
32. Xing, Y.; Zhao, C.; Li, Z.; Zhang, Y.; Li, L.; Wang, F.Y.; Wang, X.; Wang, Y.; Su, Y.; Cao, D. A Right-of-Way Based Strategy to Implement Safe and Efficient Driving at Non-Signalized Intersections for Automated Vehicles. arXiv 2019, arXiv:1905.01150.

33. Yudin, D.A.; Skrynnik, A.; Krishtopik, A.; Belkin, I.; Panov, A.I. Object Detection with Deep Neural Networks for Reinforcement Learning in the Task of Autonomous Vehicles Path Planning at the Intersection. Opt. Mem. Neural Netw. 2019, 28, 283-295. [CrossRef]

34. Isele, D.; Rahimi, R.; Cosgun, A.; Subramanian, K.; Fujimura, K. Navigating Occluded Intersections with Autonomous Vehicles using Deep Reinforcement Learning. In Proceedings of the 2018 IEEE International Conference on Robotics and Automation (ICRA), Brisbane, QLD, Australia, 21-25 May 2018.

35. Wong, A.; Shafiee, M.J.; Siva, P.; Wang, X.Y. A Deep-Structured Fully Connected Random Field Model for Structured Inference. IEEE Access 2015, 3, 469-477. [CrossRef]

36. Olayode, O.I.; Tartibu, L.K.; Okwu, M.O. Application of Artificial Intelligence in Traffic Control System of Non-autonomous Vehicles at Signalized Road Intersection. Procedia CIRP 2020, 91, 194-200. [CrossRef]

37. Dias, C.; Iryo-Asano, M.; Abdullah, M.; Oguchi, T.; Alhajyaseen, W. Modeling Trajectories and Trajectory Variation of Turning Vehicles at Signalized Intersections. IEEE Access 2020, 8, 109821-109834. [CrossRef]

38. Wolfermann, A.; Alhajyaseen, W.K.; Nakamura, H. Modeling speed pro_les of turning vehicles at signalized intersections. In Proceedings of the 3rd International Conference on Road Safety and Simulation RSS2011, Indianapolis, IN, USA, 14-16 September 2011; Transportation Research Board TRB, 01/01/2011. pp. 1-7.

39. Flash, T.; Hogan, N. The coordination of arm movements: An experimentally confirmed mathematical model. J. Neurosci. 1985, 5, 1688-1703. [CrossRef] [PubMed]

40. Berktaş, E. Şentürk; Tanyel, S. Effect of Autonomous Vehicles on Performance of Signalized Intersections. J. Transp. Eng. Part A Syst. 2020, 146, 04019061. [CrossRef]

41. Levin, M.W.; Rey, D. Conflict-point formulation of intersection control for autonomous vehicles. Transp. Res. Part C Emerg. Technol. 2017, 85, 528-547. [CrossRef]

42. Chen, F.; Song, M.; Ma, X.; Zhu, X. Assess the impacts of different autonomous trucks' lateral control modes on asphalt pavement performance. Transp. Res. Part C Emerg. Technol. 2019, 103, 17-29. [CrossRef]

43. Gołębiowski, P.; Gołda, I.J.; Izdebski, M.; Kłodawski, M.; Jachimowski, R.; Szczepański, E. The evaluation of the sustainable transport system development with the scenario analyses procedure. J. Vibroeng. 2017, 19, 5627-5638. [CrossRef]

44. Gungor, O.E.; Al-Qadi, I.L. Wander 2D: A flexible pavement design framework for autonomous and connected trucks. Int. J. Pavement Eng. 2020, 1-16. [CrossRef]

45. Jacyna, M.; Wasiak, M.; Kłodawski, M.; Lewczuk, K. Simulation model of transport system of poland as a tool for developing sustainable transport. Arch. Transp. 2014, 31, 23-35. [CrossRef]

46. Steyn, W.J.V.; Maina, J.W. Guidelines for the use of accelerated pavement testing data in autonomous vehicle infrastructure research. J. Traffic Transp. Eng. (Engl. Ed.) 2019, 6, 273-281. [CrossRef]

47. Tesoriere, G.; Canale, A.; Severino, A.; Mrak, I.; Campisi, T. The management of pedestrian emergency through dynamic assignment: Some consideration about the "Refugee Hellenism" Square of Kalamaria (Greece). AIP Conf. Proc. 2019, $2186,160004$.

48. Arena, F.; Pau, G.; Severino, A. An Overview on the Current Status and Future Perspectives of Smart Cars. Infrastructures 2020, 5, 53. [CrossRef] 